\title{
Dosimetric Effects of a Commercial Breast Board Patient Support System
}

\author{
Eve Schodowski, Austin Wilkinson, Sadik Khuder, David Pearson* \\ Department of Radiation Oncology, University of Toledo, Toledo, USA \\ Email: ^dpearson@physics.utoledo.edu
}

How to cite this paper: Schodowski, E., Wilkinson, A., Khuder, S. and Pearson, D. (2022) Dosimetric Effects of a Commercial Breast Board Patient Support System. International Journal of Medical Physics, Clinical Engineering and Radiation Oncology, 11, $1-11$.

https://doi.org/10.4236/ijmpcero.2022.111001

Received: November 8, 2021

Accepted: December 24, 2021

Published: December 27, 2021

Copyright $\odot 2022$ by author(s) and Scientific Research Publishing Inc. This work is licensed under the Creative Commons Attribution International License (CC BY 4.0).

http://creativecommons.org/licenses/by/4.0/

(c) (i) Open Access

\begin{abstract}
Aim: In prone breast treatments, a carbon fiber support device resides under the contralateral breast. Tangent beams are designed to encompass the treated breast and these often pass through the board at a shallow angle, resulting in significant attenuation. Our planners account for this attenuation by adding field-in-field dose to the deep part of the breast, through the board. Concern was raised about how accurate the treatment delivery is when the inherent uncertainties of patients' positions are accounted for. Furthermore, transmission measurements are usually carried out perpendicular to the board, a non-clinical situation. The goal of this study is to evaluate the dosimetric effect of the board and the robustness of the plan to positional uncertainty. Materials and Methods: Twenty-two breast patients treated on a commercial prone breast board between 2017 and 2020 were selected for this retrospective study. To evaluate the board's attenuation, we compared the plans with the board removed from the dose calculation. To quantify the robustness of this technique, we moved the beam isocenter with respect to the patient and board. Results: Our results showed that when the breast board is removed from a plan which was designed to account for the board attenuation, the average point dose increases by $7.48 \%$, with a maximum of $22 \%$. Comparing results with a mixed Analysis of Variance (ANOVA) and a least-square means analysis, our robustness evaluation indicates that anterior shifts at every magnitude $(1 \mathrm{~mm}$ through $5 \mathrm{~mm})$ make a significant difference in all dose statistics (D95, max, 95\% prescription coverage and homogeneity index) investigated. In/out and right/left shifts resulted in an insignificant change in dose statistics. Conclusion: Prone breast boards can add significant dosimetric uncertainty into the treatment delivery process. Accounting for plan robustness in the design of the plan is highly recommended. A prone breast board design with support moved away from the beam path is warranted.
\end{abstract}

\section{Keywords}

Prone Breast Treatment, Breast Board, Attenuating Structure, Interfractional 
Set-Up, Patient Support System, Robustness

\section{Introduction}

The introduction of prone breast treatment in the US stems from an investigation into an alternative technique for irradiating the breast following breast-conserving surgery. The intent was to improve the dose homogeneity throughout the breast and reduce the dose to the lungs and heart [1]. In prone breast treatments, an immobilization device is utilized which allows patients to be treated reproducibly. This prone breast support system, commonly referred to as a prone breast board, includes a carbon fiber support device which resides under the contralateral breast. Typically, tangent beams are used to deliver a uniform dose to the ipsilateral breast [1]-[9] as it is a simple planning technique to design and treat, although intensity modulation has also been successfully used [10] [11]. These tangent beams often pass through the prone breast board, resulting in beam attenuation dependent on the angle of the beam with respect to the board (Figure 1). This can be corrected using field-in-field techniques. Additionally, considerable setup errors have been shown to exist when treating prone breast patients due to the challenge of reproducibly positioning the patient [12]. Kirby et al. showed that setup errors in a population of breast patients treated prone $(3.1-4.3 \mathrm{~mm})$ were over twice as great as when they were treated supine (1.3 $1.9 \mathrm{~mm}$ ). Thus, the tangents designed to account for board attenuation may be displaced relative to the patient and the board. Finally, AAPM (American Association of Physicists in Medicine) Task Group 176 studied the effects of table tops and ancillary devices on the dose calculations of treatment plans. Prone breast boards were not implicitly included although one can attempt to apply the general recommendations to this case. They recommended primarily avoiding immobilization devices but that is not possible in the case of tangents and this prone breast board. When it is not possible to avoid the immobilization device then, according to TG176, it should be accounted for in the dose calculation. As we will see, it is accounting for the attenuation that poses the difficulty with this technique.

The goals of this study are to evaluate the dosimetric effect of the commercially available prone breast board used in our clinic and to investigate prone breast plan robustness. Therefore, in this paper, we quantify the board attenuation and the robustness of tangent breast plans that were used clinically on a group of previously treated patients $(n=22)$.

\section{Materials and Methods}

\subsection{Dosimetric Effect of the Breast Board}

Tangent beams are designed to encompass the treated breast and often pass through the board at a shallow angle, resulting in significant attenuation. Our 


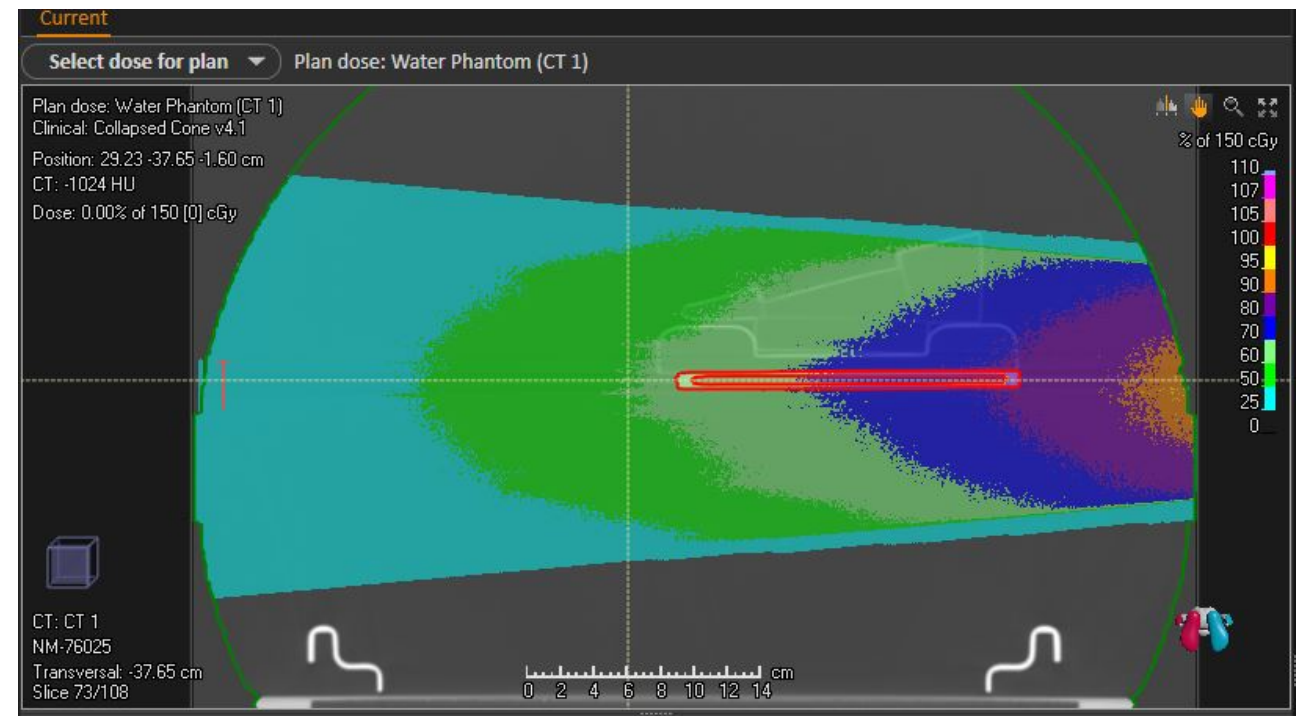

(A)

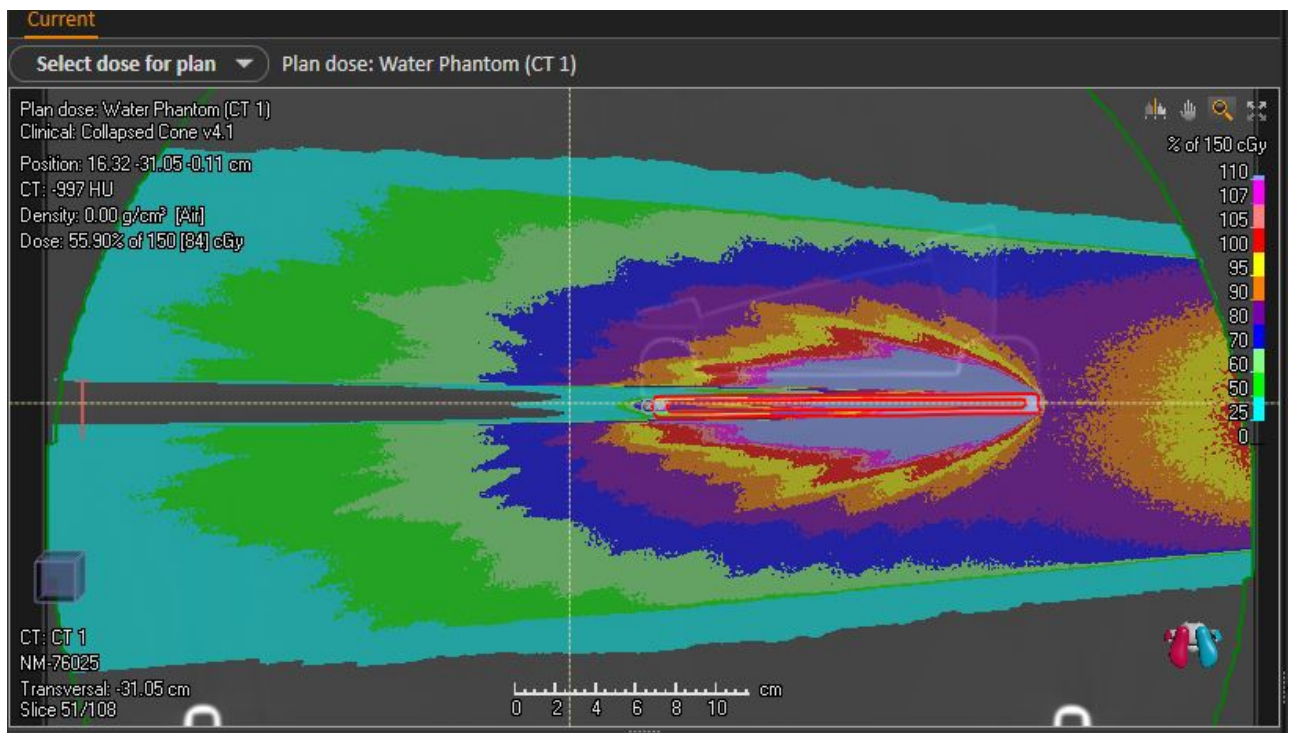

(B)

Figure 1. The breast board as an attenuating structure. (A) Dose distribution of an open field with no attenuating structures. The breast board is outlined in red and was not included in any dose calculation. In this figure the board is for aesthetic purposes only; (B) Dose distribution of an open field with the breast board as an attenuating structure. A sharp reduction in dose can be seen leaving the board, creating an area of reduced coverage; this is the "board shadow".

planners account for this attenuation by adding field-in-field dose to the deep part of the breast, through the board. Concern was raised about the accuracy of the treatment delivery when the inherent uncertainties of patient position are accounted for (setup error). The goal of this study is to evaluate the dosimetric effect of board and the robustness of the plan to positional uncertainty. Twenty-two prone breast patients treated at the Dana Cancer Center between September 2017 and June 2020 were selected for this retrospective study. No patients were excluded. All patients received 4256 cGy in 16 fractions. Patient treatment plans 
were designed in RayStation 8A (RaySearch Laboratories, Stockholm, Sweden) which uses the Collapsed Cone Convolution algorithm to calculate photon dose. The specific board studied was the Bionix Prone Breast System (Bionix, Toledo, OH). Treatment delivery utilized a Varian TrueBeam (Varian Medical Systems, Palo Alto, CA) with a carbon fiber couch capable of translational motion and axial rotation.

Using RayStation, treatment plans were evaluated with and without the prone breast board to assess changes in dose attenuation, dose homogeneity, and prescription coverage. The treatment plans were created using the material override method, where the breast board shell and interior were set as support structure Regions of Interest (ROI). The board shell and interior were then contoured and overridden to carbon fiber and air, respectively.

To compare treatment plans with and without the breast board, a new ROI based on dose was created. This ROI was defined as 95\% of the 4256 cGy Planning Treatment Volume (PTV), calculated from the dose in the clinically delivered treatment plan which was designed to account for the attenuation of the prone breast board. The breast board ROI of carbon fiber and air was then removed from the treatment plan and the dose was recalculated, but not rescaled, so that the monitor units (MUs) remained the same. Shown below is the dosimetric effect on the isodose lines of an example clinical plan where a field-in-field technique was used to account for the carbon fiber prone breast board (left). On the right, the board has been removed from that same plan and the dose recalculated with the same MUs. This shows the significance of the attenuating properties of the board and the difficulties faced by our planners to accurately account for it.

The board is often in the direct path of, and almost parallel to, the beam which creates the unique problem of a "board shadow" being cast behind it (Figure 1 and Figure 2). This board shadow is an area of reduced coverage, specifically in the area near the chest wall. To counter this, we utilize field-in-field techniques with segments specifically aimed at the board, to bring the coverage back up so that 4256 cGy covers $95 \%$ of the physicians PTV.

Beam attenuation of a device such as this prone breast board can be measured, as recommended by AAPM Task Group 176 [13]. However, since only a small fraction of the tangents beams traverses the support structure a transmission factor cannot be applied to the beam as a whole. Treatment plans at our institution were designed accounting for the board and its dose attenuation by including the structure in the external contour. Thus, removal of the breast board from treatment plans resulted in an area of overcompensated prescription coverage in the shadow of the board. To quantify the board's attenuation in this clinical geometry, a Point of Interest (POI) approximately in the middle of the shadow of the board was selected and compared between treatment plans.

ROI and POI dose statistics were calculated and recorded for the treatment plan with and without the breast board. Further, prescription coverage, defined 

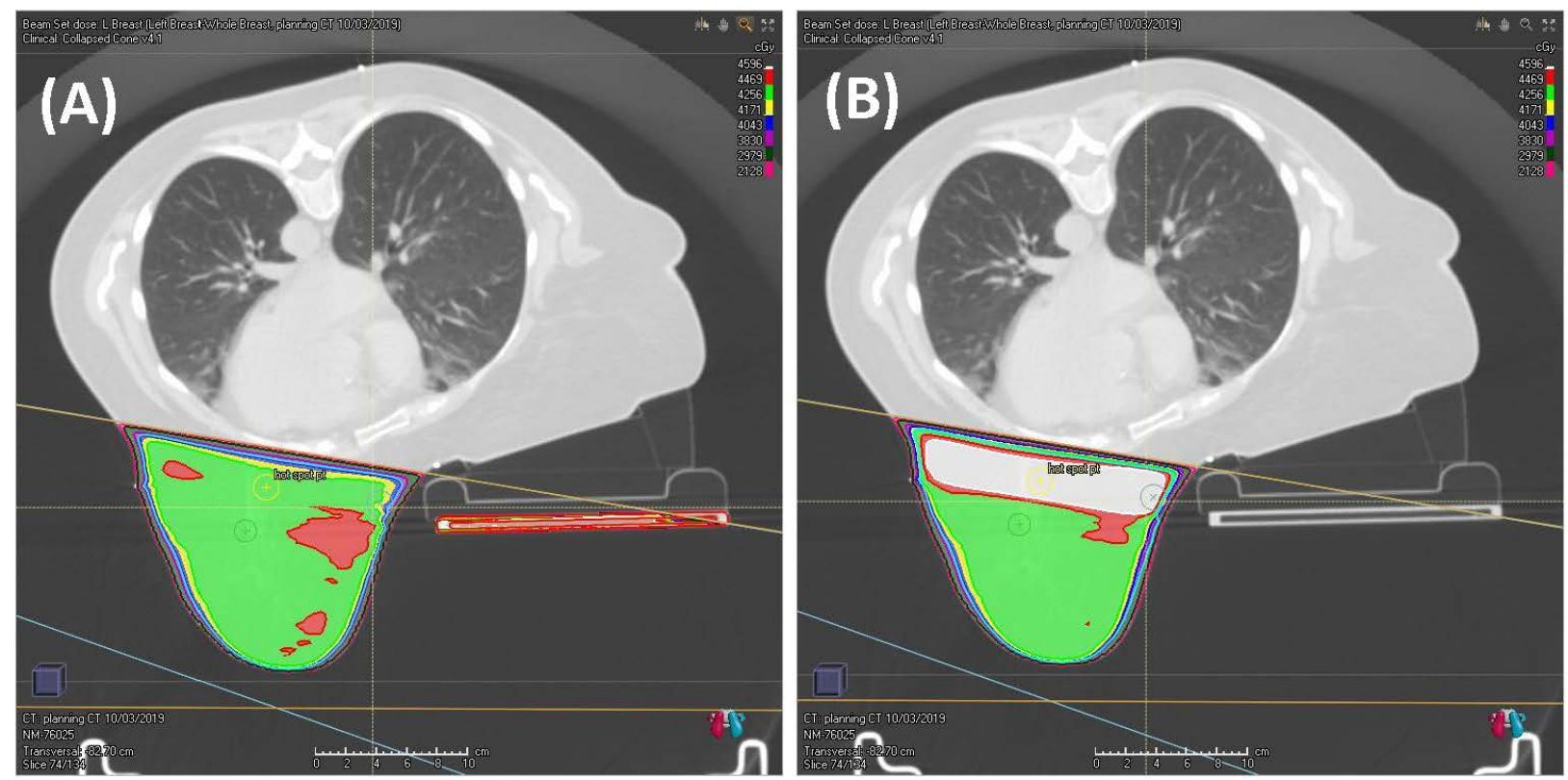

Figure 2. The isodose lines showing the dosimetric effect of removing the prone breast board from a typical clinical plan. (A) shows the planned dose distribution with the board included; (B) shows the dosimetric effect of removing the board. The isodose lines of note are green (100\%), red (105\%) and white (108\% of prescription dose). The prone breast board support is contoured as a red ROI. The ROI has a carbon fiber outer shell and air within.

as at least $95 \%$ volume of the PTV, was calculated and recorded for comparison. Additionally, the length of the breast board in the path of the beam was determined at each plan's isocenter. This measurement was made as we hypothesized percentage of the breast board in the path of the beam would correlate to the percent change in hot spot point dose (Figure 3).

For statistical analysis, means and percent changes in hot spot point dose, homogeneity index, and prescription coverage were calculated and tabulated (Table 1). The homogeneity index was defined as D98\% divided by D2\%. These data sets were used to calculate descriptive statistics using the Microsoft Excel (Microsoft Corporation, Redmond, WA) add-in Analysis ToolPak. Specifically, a paired two sample t-test was used to determine significance between dose parameters ( $\mathrm{Ta}$ ble 1). Further, a linear regression was calculated to investigate correlation between percent of the board in the beam path and percent change in hot spot point dose (Figure 3).

\subsection{Robustness and Setup Uncertainty}

Inter-fractional setup uncertainty and robustness of prone treatment plans were investigated. Robustness is a treatment plan's resistance to change when introduced with a motion or set up error. We hypothesized that the planning technique of using field-in-field to produce a homogeneous dose distribution in the shadow of the board would give rise to plans that would not be robust to the inherent set up uncertainty of prone breast treatments. We made this hypothesis as the field segments aimed at the board in the treatment plan would need to align precisely to the board at every treatment. 


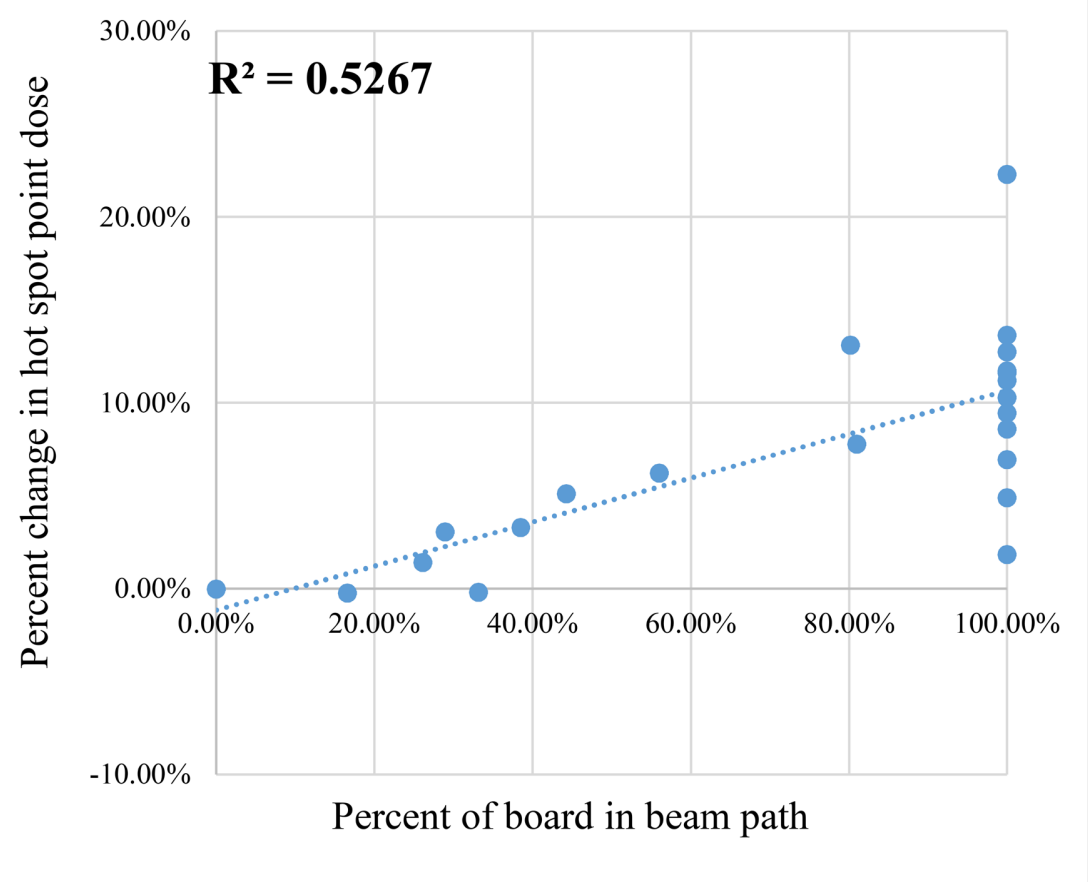

Figure 3. Percent of board in beam path vs change in hot spot point dose. A moderate correlation between percent of board in beam path and change in hot spot point dose was found with an $\mathrm{R}$ square value of 0.5267 (ANOVA significance $\mathrm{F}=0.0001$, obtained from Analysis ToolPak in Excel).

Table 1. Significance of attenuation of the board to treatment planning, comparing plans with and without breast board.

\begin{tabular}{cccc}
\hline Treatment plan & $\begin{array}{c}\text { Mean of max } \\
\text { point dose } \\
(\mathrm{cGy})\end{array}$ & $\begin{array}{c}\text { Mean 95\% } \\
\text { prescription } \\
\text { coverage }\end{array}$ & $\begin{array}{c}\text { Mean dose } \\
\text { homogeneity } \\
(\mathrm{D} 98 \% / \mathrm{D} 2 \%)\end{array}$ \\
\hline With breast board & $4470 \pm 116$ & $98.77 \% \pm 0.32 \%$ & $0.8829 \pm 0.0187$ \\
$\begin{array}{c}\text { Without breast board } \\
\begin{array}{c}\text { Percent change when } \\
\text { board is unaccounted }\end{array}\end{array}$ & $7849 \pm 327$ & $98.50 \% \pm 0.95 \%$ & $0.8299 \pm 0.0571$ \\
\hline Value & 0.00001 & $-0.29 \% \pm 0.92 \%$ & $-5.30 \% \pm 4.75 \%$ \\
\hline
\end{tabular}

Prior studies show that setup errors were likely to occur and our own imaging confirmed that the patients' positions on the board each day can vary [11] [12]. The therapists' main focus is to align the beam to the patient's anatomy and not to the board. The dose statistics used to assess each plan's robustness were D95\%, prescription coverage, homogeneity index, and maximum point dose. Maximum point dose was defined as the greatest dose at 0.035 Cubic Centimeters (CC) volume in the PTV. These parameters were tested using the "perturbed dose" feature in RayStation, which shifts a plan's isocenter relative to the patient CT dataset and therefore simulates setup error. For each patient in the study, the isocenter was perturbed to $5 \mathrm{~mm}$ and $-5 \mathrm{~mm}$ in $1 \mathrm{~mm}$ increments, with the dose 
statistics being recorded for each increment. The isocenter of each patient was perturbed in the positive and negative $\mathrm{X}, \mathrm{Y}$, and $\mathrm{Z}$ axes, corresponding to perturbations in the left/right, superior/inferior, and anterior/posterior orientations of the patient, respectively. The dose statistics for each perturbation were compared using a mixed ANVOA and least square means calculation on the software SAS 9.4 (SAS Institute, Cary, North Carolina). Figures 4(A)-(C) summarizes findings.

\section{Results}

There is a statistically significant difference (bolded) in hot spot max point dose and dose homogeneity but not for $95 \%$ prescription coverage $(\alpha=0.05)$. When
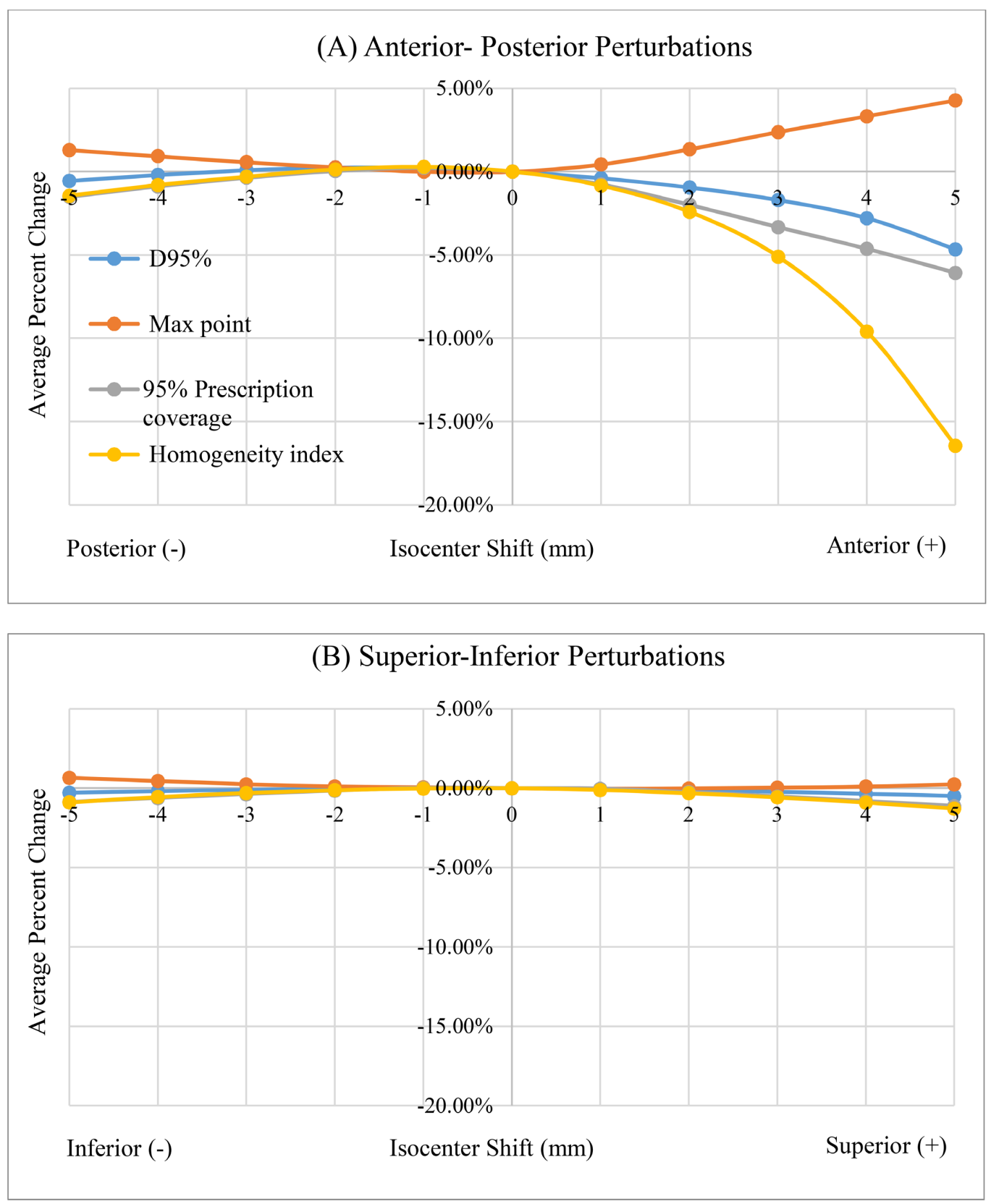


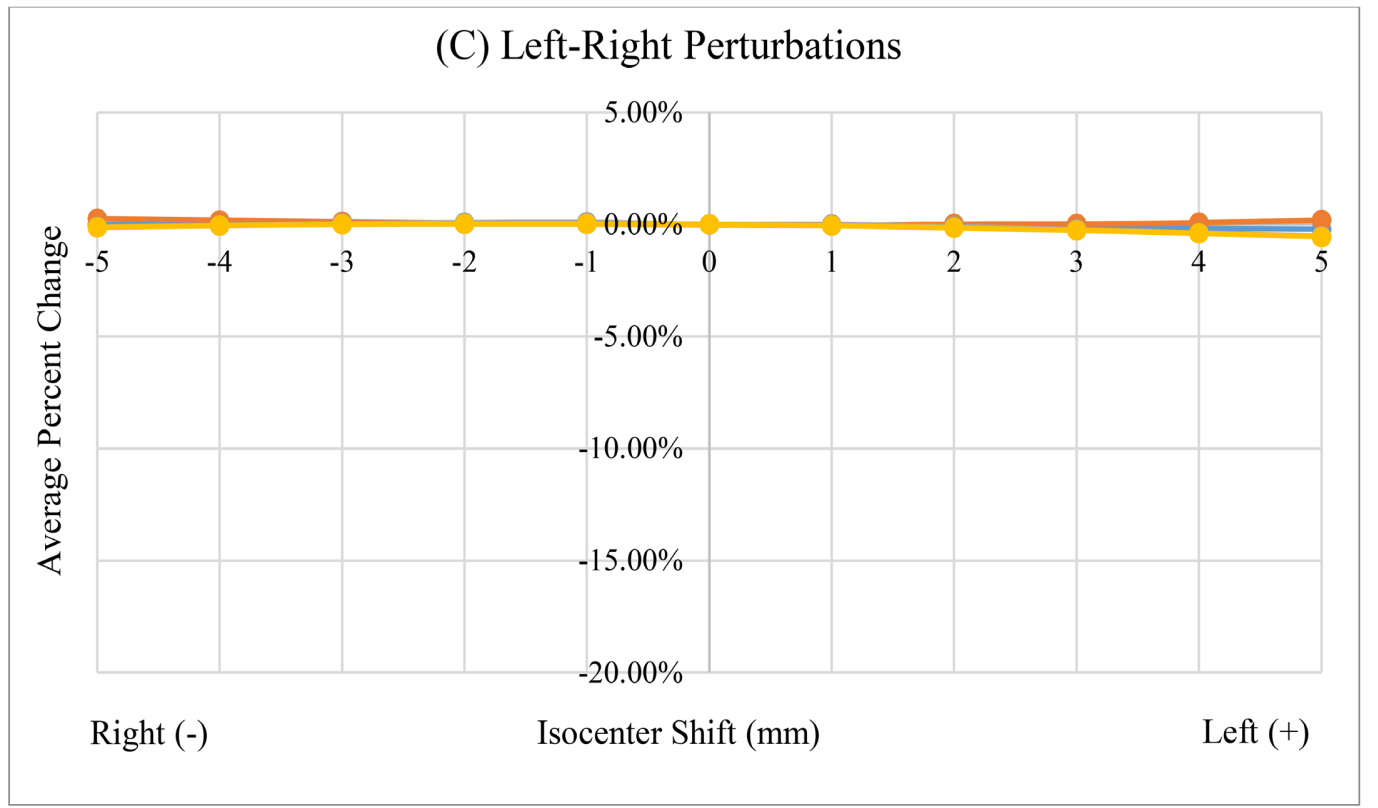

Figure 4. Treatment plan isocenter perturbations, simulating inter-fractional set up error. Mixed ANOVA and least square means analysis indicated anterior perturbations differ significantly from zero at every isocenter shift magnitude and for each dose statistic investigated $(\mathrm{p}<0.0001)(\mathrm{A})$. Occasionally, posterior per-

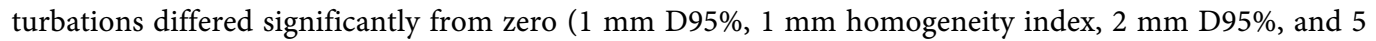
mm prescription coverage) (A). All right-left and superior-inferior perturbations suggest an insignificant change in dose statistics $((\mathrm{B})$ and $(\mathrm{C}))$.

the breast board is unaccounted for in treatment planning, on average hot spot point dose becomes $7.48 \%$ hotter, $95 \%$ prescription coverage decreases by $0.29 \%$, and the dose distribution becomes $5.30 \%$ less homogenous.

Table 1 shows the attenuating effects of the prone breast board. The attenuation, as determined on a patient by patient basis and accounting for plan geometry, was on average $7.48 \%$ with a maximum for one patient of $22 \%$. The variation in attenuation was hypothesized to be due to the lateral length of the carbon fiber that supports the contralateral breast being in the tangent beams. In cases where the tangent angle is shallow, the opposing tangents would be almost parallel to the board, resulting in the greatest length of board in the path of the beam and the largest attenuation. To investigate this, we measured the length of the board in the path of the beam, visible on the planning CT. This is shown in Figure 4. In many cases, the design of the tangents that optimized breast coverage while minimizing the dose to critical structures resulted in $100 \%$ of the carbon fiber support (outlined in red in Figure 1) being in the path of the beam. However, the attenuation for such patients varied from less than $5 \%$ to over $20 \%$, suggesting more complex geometries may come into effect.

The plan robustness results, as shown in Figure 4(A), indicate that large changes in plan quality metrics can occur with only small changes (a few $\mathrm{mm}$ ) in the anterior-posterior position of the of the beam isocenter relative to the patient and, more critically in this case, the carbon fiber support. Changes in patient position in the cranial, caudal, and lateral directions resulted in little dosimetric effect sug- 
gesting plans are relatively robust to set up uncertainties in these directions.

\section{Discussion}

In this study, we investigated prone breast board attenuation and prone breast treatment plan robustness for a commercially available Bionix breast support system. This was done by retrospectively reviewing treatment plans with the breast board accounted for and then calculating the same plan without the breast board or, in the case of robustness, with an isocenter different from that planned. We then compared dose statistics between plans.

Regarding dose attenuation of the prone breast board, our results suggest there is a significant difference in the dose distribution and dose homogeneity, but not for $95 \%$ prescription coverage, when comparing plans designed with and calculated without the breast board. When the breast board is removed from a plan which was designed to account for the board attenuation, on average the point dose increases by $7.48 \%$ and dose distribution became $5.30 \%$ less homogenous (Table 1 ). We hypothesized the difference in dose attenuation may be due to the amount of the breast board obstructing the path of the beam, which would then correlate to percent change in the maximum point dose. After determining the length of the board in the path of the beam at each plan's isocenter, we found a moderate correlation between percent of board in beam path and change in max point dose with an R square value of 0.5267 (Figure 3). These results suggest that attenuation is likely dependent on a number of factors and not just the length of carbon fiber support in the tangent beams.

Overall, our data suggest that failing to account for the attenuating effects of the prone breast board would underdose the patient in the deep aspect of the breast by up to $22 \%$ or, $7.48 \%$, on average. Note that this attenuation is $5 \%$ worse than any immobilization device listed in TG-176, suggesting that the design of this breast board is not consistent, in terms of attenuation factors, with the devices studied by that group.

We investigated treatment plan robustness by shifting the patients CT data set relevant to the isocenter along the $\mathrm{X}, \mathrm{Y}$, and $\mathrm{Z}$ axes, simulating inter-fractional set up error or movement of the patient in anterior/posterior, left/right and in/out directions, in $1 \mathrm{~mm}$ increments up to $5 \mathrm{~mm}$. After comparing results with a mixed ANOVA and least square means analysis, our data indicates that even small anterior shifts make a significant difference in all dose statistics (D95, max, 95\% prescription coverage and homogeneity index) investigated. Infrequently, posterior shifts caused significant differences (D95\% at $1 \mathrm{~mm}$, homogeneity index at $1 \mathrm{~mm}$, D95\% at $2 \mathrm{~mm}$, and prescription coverage at $5 \mathrm{~mm}$ ). Conversely, all right/left and in/out shifts investigated resulted in an insignificant change in dose statistics, warranting further investigation.

Finally, limitations of this study include the modest sample size of twenty-two patients and potentials for human error in creating the study's regions of interest when manually contouring ROIs in RayStation. Future studies could aim to cla- 
rify trends in data by increasing sample size and by standardizing a method for designing regions of interest in RayStation.

\section{Conclusion}

In conclusion, this study aimed to investigate and quantify prone breast board attenuation and prone breast treatment plan robustness. Overall, this study suggests the radiation oncology team should be cautious when designing prone breast treatment plans by accounting for the board's potential attenuation. Furthermore, therapists should be especially cautious of patient perturbations in the anterior or posterior directions during inter-fractional setup. Manufacturers of prone breast patient support systems should be mindful of the dosimetric effects caused by the board design. Although the authors recognize the need for a system that is not easily damaged and is comfortable for patients, the materials and their geometries, which attenuate the beam by up to $22 \%$, should be avoided.

\section{Conflicts of Interest}

The authors declare no conflicts of interest regarding the publication of this paper.

\section{References}

[1] Merchant, T.E. and McCormick, B. (1994) Prone Position Breast Irradiation. International Journal of Radiation Oncology · Biology · Physics, 30, 197-203.

[2] Algan, Ö. Fowble, B., McNeeley, S. and Fein, D. (1998) Use of the Prone Position in Radiation Treatment for Women with Early Stage Breast Cancer. International Journal of Radiation Oncology · Biology · Physics, 40, 1137-1140.

[3] Grann, A., McCormick, B., Chabner, E.S., Gollamudi, S.V., Schupak, K.D., Mychalczak, B.R., Heerdt, A.S., Merchant, T.E. and Hunt, M.A. (2000) Prone Breast Radiotherapy in Early-Stage Breast Cancer: A Preliminary Analysis. International Journal of Radiation Oncology $\cdot$ Biology $\cdot$ Physics, 47, 319-325.

[4] Buijsen, J., Jager, J.J., Bovendeerd, J., Voncken, R., Borger, J.H., Boersma, L.J., Murrer, L.H.P. and Lambin, P. (2007) Prone Breast Irradiation for Pendulous Breasts. Radiotherapy \& Oncology, 82, 337-340. https://doi.org/10.1016/j.radonc.2006.08.014

[5] Chino, J.P. and Marks, L.B. (2008) Prone Positioning Causes the Heart to Be Displaced Anteriorly within the Thorax: Implications for Breast Cancer Treatment. International Journal of Radiation Oncology · Biology · Physics, 70, 916-920.

[6] Griem, K.L., Fetherston, P., Kuznetsova, M., Foster, G.S., Shott, S. and Chu, J. (2003) Three-Dimensional Photon Dosimetry: A Comparison of Treatment of the Intact Breast in the Supine and Prone Position. International Journal of Radiation Oncology - Biology Physics, 57, 891-899.

[7] Stegman, L.D., Beal, K.P., Hunt, M.A., Fornier, M.N. and McCormick, B. (2007) Long-Term Clinical Outcomes of Whole-Breast Irradiation Delivered in the Prone Position. International Journal of Radiation Oncology · Biology · Physics, 68, 73-81.

[8] Ramella, S. EdyIppolito, L.T., Ippolito, E., Fiore, M., Cellini, F., Stimato, G., Gaudino, D., Greco, C., Ramponi, S. and Cammilluzzi, E., Cesarini, C., Piermattei, A., Cesario, A. and D'Angelillo, R.M. (2012) Whole-Breast Irradiation: A Subgroup Analysis of Criteria to Stratify for Prone Position Treatment. Medical Dosimetry, 37, 186-191. 
https://doi.org/10.1016/j.meddos.2011.06.010

[9] Huppert, N., Jozsef, G., DeWyngaert, K., Formenti, S.C. (2011) The Role of a Prone Setup in Breast Radiation Therapy. Frontiers in Oncology, 1, 31.

https://doi.org/10.3389/fonc.2011.00031

[10] Goodmn, K.A., Hong, L., Wagman, R., Hunt, et al. (2004) Dosimetric Analysis of a Simplified Intensity Modulation Technique for Prone Breast Radiotherapy. International Journal of Radiation Oncology · Biology · Physics, 60, 95-102.

[11] Veldeman, L., De Gersem, W., Speleers, B., et al. (2012) Alternated Prone and Supine Whole-Breast Irradiation Using IMRT: Setup Precision, Respiratory Movement and Treatment Time. International Journal of Radiation Oncology $\cdot$ Biology $\cdot$ Physics, 82, 2055-2064.

[12] Kirby, A.M., Philips, M.E., Heyler, S.J., Donovan, E.M., Convery, H.M. and Yarnold, J.R. (2011) A Randomised Trial of Supine versus Prone Breast Radiotherapy (SuPr Study): Comparing Set-Up Errors and Respiratory Motion. Radiotherapy \& Oncology, 100, 221-226. https://doi.org/10.1016/j.radonc.2010.11.005

[13] Olch, A.J., Greig, L., Li, H., Mihaylov, I. and Morgan, A. (2014) Dosimetric Effects Caused by Couch Tops and Immobilization Devices: Report of AAPM Task Group 176. Medical Physics, 41, Article No. 061501. https://doi.org/10.1118/1.4876299 